\title{
Exact enumeration and Monte Carlo results for self-avoiding walks in a slab
}

\author{
R Martin ${ }^{1}$, E Orlandini' ${ }^{2}$ A L Owczarek ${ }^{3}$, A Rechnitzer ${ }^{4}$ \\ and S G Whittington ${ }^{1}$ \\ ${ }^{1}$ Department of Chemistry, University of Toronto, Toronto, Canada M5S 3H6 \\ ${ }^{2}$ Dipartimento di Fisica e Sezione INFN, Università di Padova, Padova, Italy \\ ${ }^{3}$ Department of Mathematics and Statistics, The University of Melbourne, \\ Parkville, Victoria 3010, Australia \\ ${ }^{4}$ Department of Mathematics, University of British Columbia, Vancouver, \\ Canada V6T 1 Z2
}

\begin{abstract}
.
We analyse exact enumeration data and Monte Carlo simulation results for a self-avoiding walk model of a polymer confined between two parallel attractive walls (plates). We use the exact enumeration data to establish the regions where the polymer exerts an effective attractive force between the plates and where the polymer exerts an effective repulsive force by estimating the boundary (zero-force) curve. While the phase boundaries of the phase diagram have previously been conjectured we delineate this further by establishing the order of the phase transitions for the so-called "infinite-slab" (that is, when the plates are a macroscopic distance apart). We conclude that the adsorption transitions associated with either plate are similar in nature to the half-space situation even when a polymer is attached to the opposite wall. The transition between the two adsorbed phases is established as first-order. Importantly, we conjecture a scaling theory valid in the desorbed and critically adsorbed regions of the phase diagram and demonstrate the consistency of the Monte Carlo data with these hypothesises by estimating the corresponding scaling functions.
\end{abstract}




\section{Introduction}

The effects of geometrical constraints on the thermodynamic properties of polymers are important for a variety of reasons. Because of the ability to micromanipulate individual polymer molecules using AFM or optical tweezers (see for instance [1]) there is considerable interest in the response of polymers to an applied force. In addition such constrained systems are crude models for steric stabilization and sensitized flocculation of colloidal dispersions [2].

Polymers between two parallel confining planes, where the monomers can interact with the two planes, have been studied since the seminal work on a random walk model by DiMarzio and Rubin [3]. A directed walk model closely related to Dyck paths was investigated by Brak et al [4] where the phase diagram was worked out for infinite walks between two parallel lines where the distance between the lines is very large. Depending on the interaction parameters with the two lines the phase diagram has regions of long range repulsion, short range repulsion and short range attraction. The corresponding self-avoiding walk model was investigated by Wall et al [5] who determined exact values for the entropy in two dimensions when the confining lines are very close together (see also [6, 7]). Stilck and Machado have studied the attractive walls case in two dimensions using transfer matrix techniques [8]. See also [9]. The phase diagram for the self-avoiding walk model was investigated numerically by Janse van Rensburg et al [10] and bounds on the region where the force is repulsive were determined rigorously [11]. In this paper we report a numerical study of the phase diagram for the self-avoiding walk model, using a mixture of exact enumeration and series analysis, and Monte Carlo techniques. This is an extension of the work described in [10]. In particular we focus on locating the zero force curve and on establishing the existence of regions of long range repulsion, short range repulsion and short range attraction in the phase diagram. We also conjecture a scaling theory valid in the desorbed and critically adsorbed regions of the phase diagram. We then test these conjectures with Monte Carlo data and consequently estimate finite-size scaling functions in these regions.

Consider the simple cubic lattice with coordinate system $(x, y, z)$ so that each vertex has integer coordinates. Consider $n$-edge self-avoiding walks starting at the origin with vertices numbered $j=0,1,2, \ldots n$ and with the $j$ th vertex having 
integer coordinates $\left(x_{j}, y_{j}, z_{j}\right)$. We shall be interested in such walks confined so that $0 \leq z_{j} \leq w$ for fixed $w$. Let the number of such walks be $c_{n}(w)$. It is known that the limit

$$
\kappa(w)=\lim _{n \rightarrow \infty} n^{-1} \log c_{n}(w)
$$

exists and that $\kappa(w)$ is strictly increasing in $w$ [12]. If we keep track of how many vertices are in each of the two planes $z=0$ and $z=w$ we can define the partition function

$$
Z_{n}(a, b ; w)=\sum_{u \geq 0} \sum_{v \geq 0} c_{n}(u+1, v ; w) a^{u} b^{v}
$$

where $c_{n}(u+1, v ; w)$ is the number of $n$-edge self-avoiding walks, starting at the origin, confined between the two planes $z=0$ and $z=w$, with $u+1$ vertices in $z=0$ and with $v$ vertices in $z=w$. We know that the limit defining the free energy

$$
\kappa(a, b ; w)=\lim _{n \rightarrow \infty} n^{-1} \log Z_{n}(a, b ; w)
$$

exists [11] and we are interested in the $w$-dependence of $\kappa(a, b ; w)$ for various values of $a$ and $b$. We would also like to know the singularities in $\kappa(a, b ; w)$ in the infinite $w$ limit since the loci of these singularities determine the phase diagram in the $(a, b)$-plane. The $w$-dependence of $\kappa(a, b ; w)$ determines if the force exerted by the walk on the confining planes is repulsive or attractive. If $\kappa(a, b ; w)$ is an increasing function of $w$ the force is repulsive while if it is a decreasing function of $w$ the force is attractive.

We know rigorously [11] that the force is repulsive if $a \leq 1$ or if $b \leq 1$ and we also know that it is repulsive if $a b \leq a_{c}$ where $a_{c}$ is the critical value for adsorption in the single surface problem. The value of $a_{c}$ is not known exactly but reliable numerical estimates are available [13].

\section{Exact enumeration and series analysis results}

We have exactly enumerated self-avoiding walks with up to 22 edges and extracted the values of $c_{n}(u+1, v ; w)$ for $n \leq 22$ and $w \leq 8$. We constructed the corresponding partition functions $Z_{n}(a, b ; w)$ defined in $(1.2)$ and estimated $\kappa(a, b ; w)$ by ratio 
analysis methods, assuming that $Z_{n}(a, b ; w)=A n^{h} \mu(a, b ; w)^{n}(1+O(1 / n))$ where $\mu(a, b ; w)=\exp [\kappa(a, b ; w)]$. That is, we defined

$$
R_{n}(a, b ; w)=\sqrt{Z_{n}(a, b ; w) / Z_{n-2}(a, b ; w)}
$$

which, under the above assumptions, behaves as

$$
R_{n}(a, b ; w)=\mu(a, b ; w)[1+B / n+o(1 / n)]
$$

Of course, we expect confluent correction terms which will upset the functional form in (2.2) but we are not concerned here with making very accurate estimates of $\mu(a, b ; w)$. We shall be content with the observed trends in the behaviour as $a, b$ and $w$ are varied.

In Figure 1 we show the ratio plots for $a=1, b=1$ and for $a=2, b=1$, for $w$ from 4 to 8 . That is, we plot $R_{n}(a, b ; w)$ against $1 / n$ and we expect the intercept to be $\mu(a, b ; w)$. For $a=1, b=1$ the ratio plots for the different values of $w$ are well separated and the values for small $w$ are below those for larger $w$, consistent with the limiting free energy being an increasing function of $w$ or, equivalently, with the force being repulsive. For $a=2, b=1$ the curves are very much closer together though the ratios for small $w$ are still below those for larger $w$, corresponding to a repulsive force. These results then allow us to infer a force which decreases like a power law for $a=1, b=1$ and exponentially for $a=2, b=1$. The power law behaviour is consistent with a scaling argument due to Daoud and de Gennes [14] and with the behaviour found for a directed walk model [4] for $a<a_{c}, b<a_{c}$. Similarly the behaviour at $a=2, b=1$ suggests an exponential decay of the force as found for a directed walk model for $a>a_{c}, b<b_{0}(a)$ where $b=b_{0}(a)$ is the zero force curve [4].

Because the $R_{n}(a, b ; w)$ curves discussed above are very close together we present the data in another way. Let $Q_{n}(a)$ be the partition function for the halfspace problem, ie for the problem of adsorption at a single impenetrable surface. Suppose that $a \geq b$. Define

$$
R_{n}^{\prime}(a, b ; w)=R_{n}(a, b ; w)-\sqrt{Q_{n}(a) / Q_{n-2}(a)} .
$$

That is, define the individual ratios relative to the values for the half-space problem, which changes the scale and separates the curves for different $w$. Values of $R_{n}^{\prime}(a, b ; w)$ 

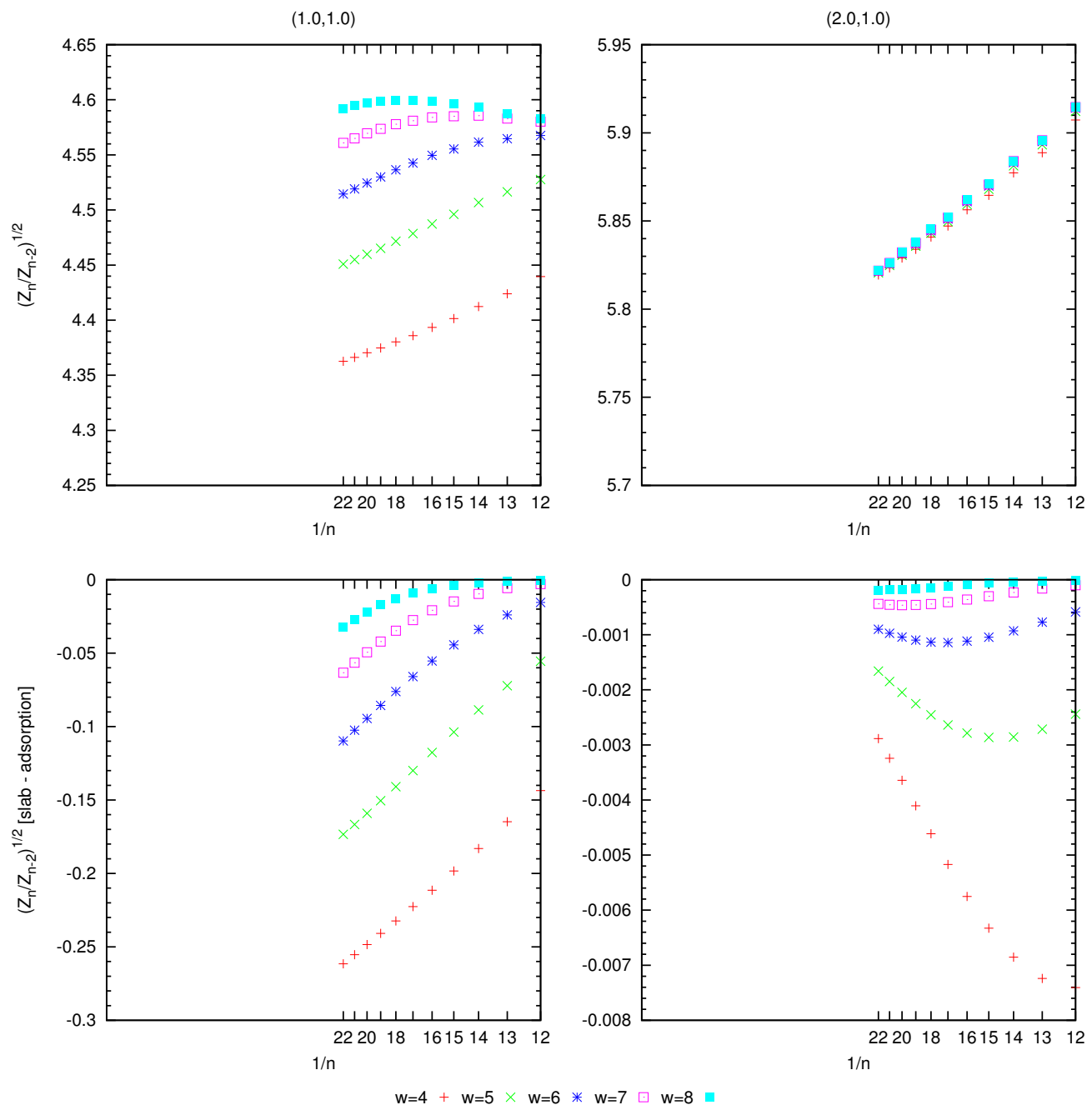

Figure 1. Ratio plots and modified ratio plots for $a=1, b=1$ and for $a=2$, $b=1$.

which are negative, and approaching zero as $w$ increases, correspond to a repulsive force. These modified ratio plots are also shown in Figure 1.

In Figure 2 we show the corresponding ratio plots for $a=2, b=2$ and for $a=3, b=2$. For $a=2, b=2$ (ie on the diagonal in the $(a, b)$-plane) the values of the ratios (and their estimated intercepts) are decreasing as $w$ increases so the force is attractive. For $a=3, b=2$ we see the same behaviour but now the ratios are very close together (ie depend only weakly on $w$ ). The values still decrease as $w$ increases 

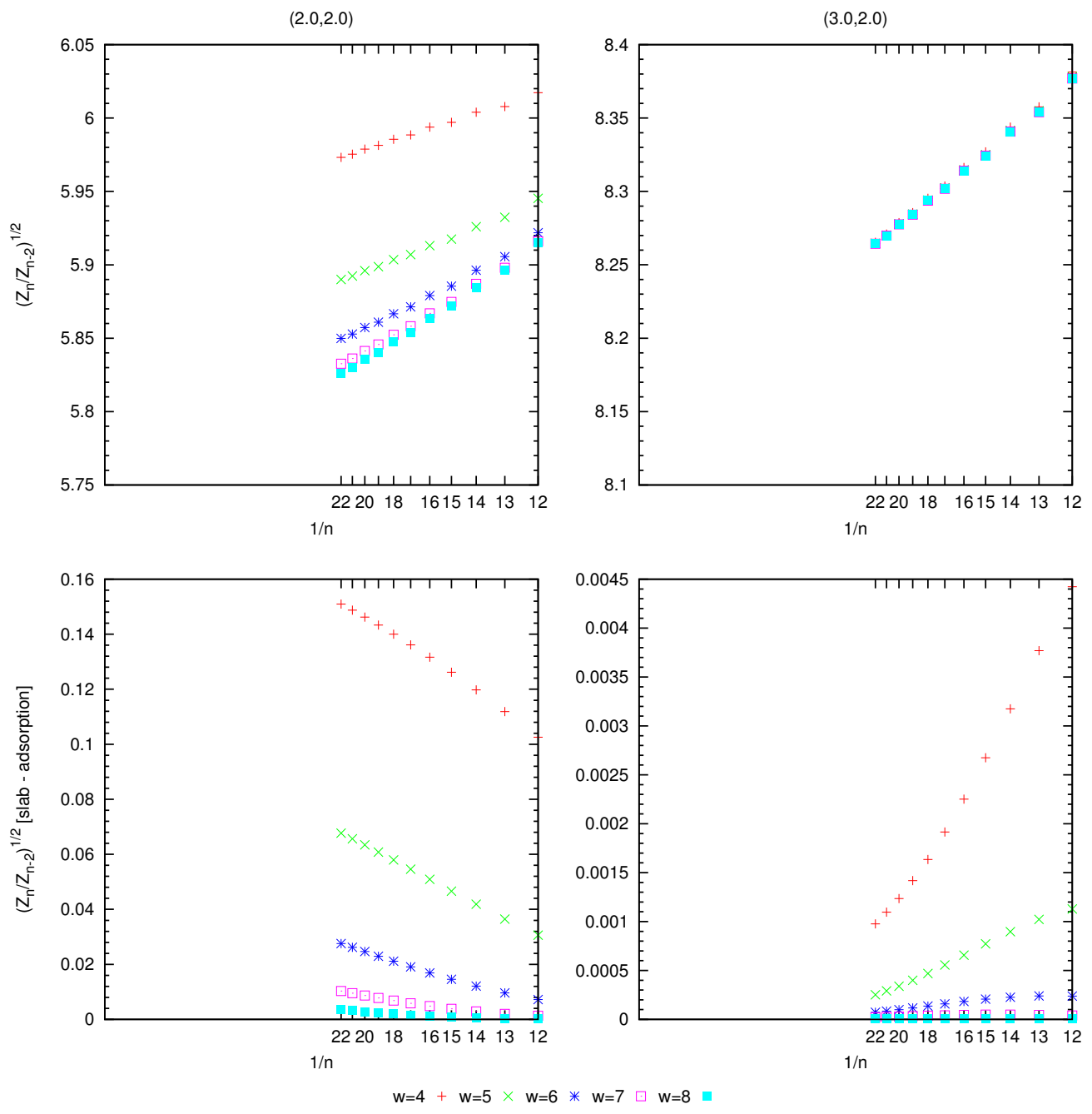

Figure 2. Ratio plots and modified ratio plots for $a=2, b=2$ and for $a=3$, $b=2$.

so the force is attractive and the weak $w$-dependence is consistent with exponential decay of the force as found for a directed walk model [4]. For the directed case the behaviour on the diagonal is predicted to be different from that elsewhere in the attractive regime, and this is seen clearly in Figure 2. The modified ratio plots (ie $R_{n}^{\prime}(a, b ; w)$ plotted against $1 / n$ at fixed values of $a$ and $b$ for a range of values of $w$ ) are also shown in Figure 2. 


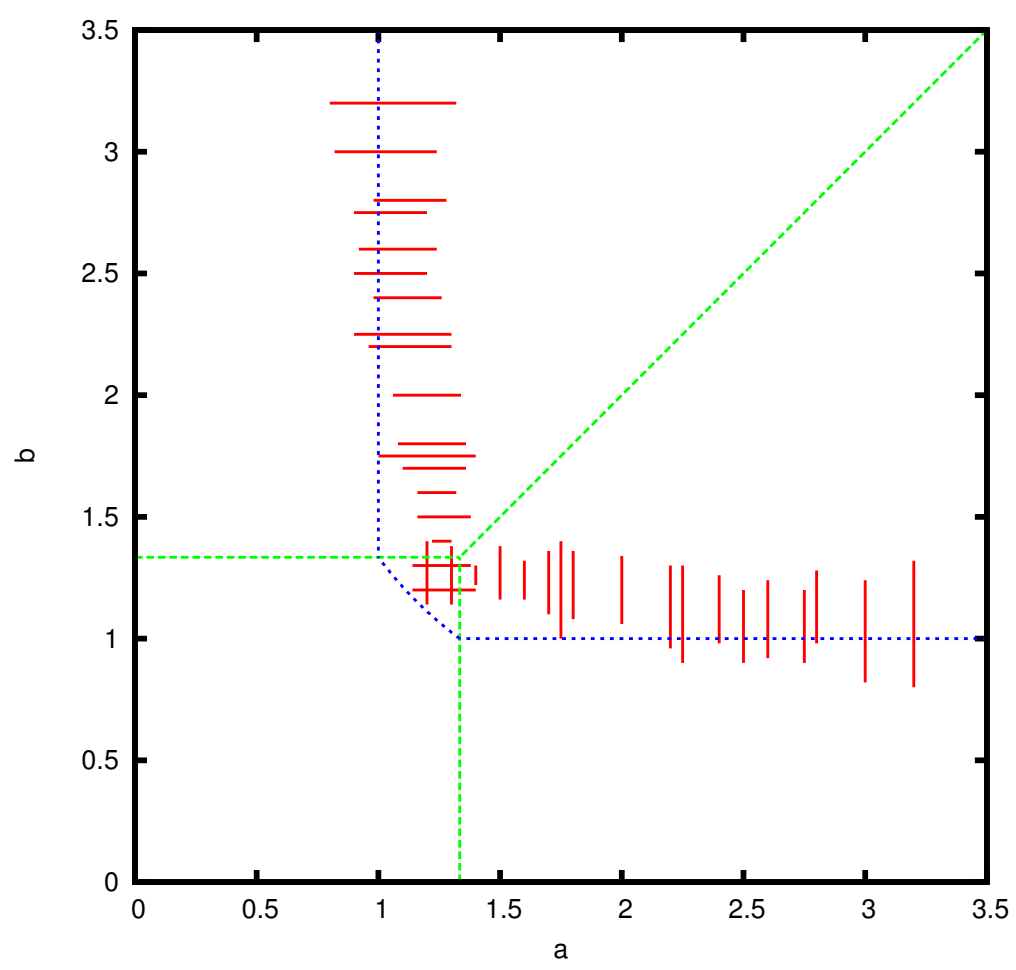

Figure 3. Estimates of the location of the zero force curve. Note that it passes through or close to the point $\left(a_{c}, a_{c}\right)$ and is asymptotic to the lines $a=1$ and $b=1$.

By repeating this type of analysis elsewhere in the $(a, b)$-plane we can identify the transition from repulsive to attractive behaviour, ie the zero force curve. Our estimate of this curve is shown in Figure 3. We also show the bound on the boundary of the repulsive regime which is known rigorously. The square with vertices $(0,0)$ and $\left(a_{c}, a_{c}\right)$, and the line $b=a$ are also shown. The estimated curve appears to have asymptotes $a=1$ and $b=1$ and the curve passes through or close to the point $\left(a_{c}, a_{c}\right)$.

\section{Scaling theory}

In this section we present scaling hypotheses for the free energy and the force between the plates in the high temperature and critical regimes $a, b \leq a_{c}$. In these regimes the force is expected to be of a power law type as a function of the width. We do not expect standard scaling arguments to hold in the low temperature regimes where 
the force is predicted to be fall off exponentially in the width.

We begin by defining the finite-size free energy $\kappa_{n}$ as

$$
\kappa_{n}(a, b ; w)=n^{-1} \log Z_{n}(a, b ; w)
$$

so that the thermodynamic limiting free energy discussed above, $\kappa(a, b ; w)$, is given by the infinite length limit given in (1.3).

There is a second double limit of the finite free energy, $\kappa_{n}(a, b ; w)$, where the length and width limits are exchanged. This more traditional case gives us the halfspace model. That is, since we are considering self-avoiding walks that are attached to the surface where the sites are weighted with the Boltzmann weight $a$ the limit of large width for fixed length is independent of the Boltzmann weight $b$. The partition function then simply becomes that of a self-avoiding walk attached to the surface in a half-space. Hence if we define

$$
\bar{\kappa}_{n}(a)=\lim _{w \rightarrow \infty} n^{-1} \log Z_{n}(a, b ; w)
$$

then the infinite length limit of $\bar{\kappa}_{n}(a)$ gives us the thermodynamic free energy, denoted $\bar{\kappa}(a)$, of the half-space.

Accordingly the standard scaling hypothesis for self-avoiding walks attached to a surface in the half-space predicts

$$
\bar{\kappa}_{n}(a) \sim \bar{\kappa}(a)+g \frac{\log n}{n}+\frac{A(a)}{n} \quad \text { as } n \rightarrow \infty .
$$

The value of $g$ depends on whether $a<a_{c}$ or $a=a_{c}$. For $a<a_{c}$ the exponent $g$ is often denoted by $\gamma_{1}-1$ and in three dimensions has been estimated as -0.32 [18]. For $a=a_{c}$ the exponent $g$ is often denoted by $\gamma_{1, s}-1$ and is only known roughly as $0.5(2)[18]$.

It has been previously proved [19] that $\bar{\kappa}(a)$, the half-space thermodynamic limit free energy, is constant for $a \leq a_{c}$ and is given by the value $\log \mu(3)$, that is, the logarithm of the growth constant for unconfined self-avoiding walks in three dimensions.

One expects that the exponent $h$, defined by the relation $Z_{n}(a, b ; w) \sim$ $A n^{h} \mu(a, b ; w)^{n}$ for the scaling of the finite slab partition function, takes on the twodimensional value of $\gamma-1$ at fixed width $w$, where $\gamma$ is the unconstrained entropic exponent, and so $h$ then is conjectured to be $11 / 32$ [20]. Note in passing that for 
$a>a_{c}$ the half-space exponent $g$ is also expected to take on this value as it is bound closely to the surface. Again, standard scaling gives

$$
\kappa_{n}(a, b ; w) \sim \kappa(a, b ; w)+h \frac{\log n}{n}+\frac{B(a, b ; w)}{n} \quad \text { as } n \rightarrow \infty
$$

Also important here is the fact that for $a, b \leq a_{c}$ one expects that $\kappa(a, b ; w) \rightarrow$ $\log \mu(3)=\bar{\kappa}(a)$ as $w \rightarrow \infty$ : this was explicitly found in the exactly solved case [4].

This fixed width scaling (3.4) can be reconciled with the infinite width scaling (3.3) using the hypothesis of a scaling function in an appropriate scaling variable. Since walks in a half-space typically extend out from the surface an amount proportional to $n^{\nu}$ where $\nu$ is the three-dimensional value of the radius of gyration exponent one can conjecture that this scaling variable should be $n^{\nu} / w$.

Hence we conjecture the scaling form of the free energy to be

$$
\kappa_{n}(a, b ; w) \sim \log \mu(3)+g \frac{\log n}{n}+\frac{1}{n} \mathcal{K}\left(d n^{\nu} / w\right) \quad \text { as } n, w \rightarrow \infty
$$

with $n^{\nu} / w$ fixed, and the scaling function $\mathcal{K}(x)$ obeying

$$
\mathcal{K}(x) \sim A(a) \quad \text { as } x \rightarrow 0
$$

and

$$
\mathcal{K}(x) \sim c x^{1 / \nu}+\frac{(h-g)}{\nu} \log (x) \quad \text { as } x \rightarrow \infty
$$

with $c$ and $d$ being generic constants here and below - note that $d$ is a nonuniversal factor. It is important to understand that the scaling function depends on whether the underlying infinite-slit system is critical or not as the temperature is varied. Hence there are four different scaling functions: one for $a, b<a_{c}$, one for $a=a_{c}, b<a_{c}$, one for $a<a_{c}, b=a_{c}$ and one for $a=a_{c}, b=a_{c}$.

We note that the conjectured scaling form is an expansion about the half-space limit. Of course it would be also reasonable to conjecture a scaling form that is an expansion about the infinite-slit, as in

$$
\kappa_{n}(a, b ; w) \sim \log \mu(3)+h \frac{\log n}{n}+\frac{1}{n} \hat{\mathcal{K}}\left(d n^{\nu} / w\right) \quad \text { as } n, w \rightarrow \infty
$$

Without going into detail we found that it was more difficult to match this two variable scaling form with the single variable scaling limits.

By using the definition of the force $F_{n}(a, b ; w)$ induced by the polymer between the plates as

$$
F_{n}(a, b ; w)=\frac{\partial \kappa_{n}(a, b ; w)}{\partial w}
$$


we can conjecture a scaling form for the force as

$$
F_{n}(a, b ; w) \sim \frac{1}{n^{(1+\nu)}} \mathcal{F}\left(d n^{\nu} / w\right) \quad \text { as } n, w \rightarrow \infty
$$

where

$$
\mathcal{F}(x) \sim c x^{1+1 / \nu} \quad \text { as } x \rightarrow \infty .
$$

Hence the force between the plates with an "infinite" length polymer $(F(a, b ; w)=$ $\left.\lim _{n \rightarrow \infty} F_{n}(a, b ; w)\right)$ between them scales as

$$
F(a, b ; w) \sim \frac{c}{w^{(1+1 / \nu)}} \quad \text { as } w \rightarrow \infty .
$$

All this is consistent with the older scaling theory [14].

\section{Monte Carlo results}

We have used FlatPERM [16] to simulate self-avoiding walks on the cubic lattice of lengths up to 512 with vertices confined to lie between two planes, one at $z=0$ and the other at $z=w$ for $w=12,16,20, \ldots, 40$. These lengths and widths were chosen so as to provide a range of values of the scaling variable $n^{\nu} / w$ of order one and centred on 1. While such system sizes prevented us from exploring the entire $(a, b)$ parameter space as was done in [10] the subsets chosen allowed the calculation of finer estimates of quantities. We have simulated these systems along four lines in the $(a, b)$ plane (described below). These simulations required memory growing with the square of the length and so larger systems could be simulated. Each run consisted of between $2 \times 10^{12}$ to $3 \times 10^{12}$ samples and took several weeks of computer time.

The expected infinite-slab phase diagram, which was schematically conjectured in [10], is displayed in Figure 4, along with the four lines along which simulations were undertaken. For $a$ and $b$ below the half-space adsorption critical point $a_{c} \approx 1.33$ (value taken from [13]) the polymer is desorbed. For $a>\max \left\{a_{c}, b\right\}$ the polymer is adsorbed to the bottom surface and for $b>\max \left\{a, b_{c}\right\}$ the polymer is adsorbed to the top surface. There is also a transition between the two adsorbed phases along the line $a=b$ for $a, b>a_{c}$. We simulated the system along the following four lines in the $(a, b)$ plane: $(1 / 2, b),(2, b),(a, 1 / 2)$ and $(a, 2)$. The lines $(1 / 2, b)$ and $(a, 1 / 2)$ were chosen to allow us to study the phase transition between the desorbed phase 


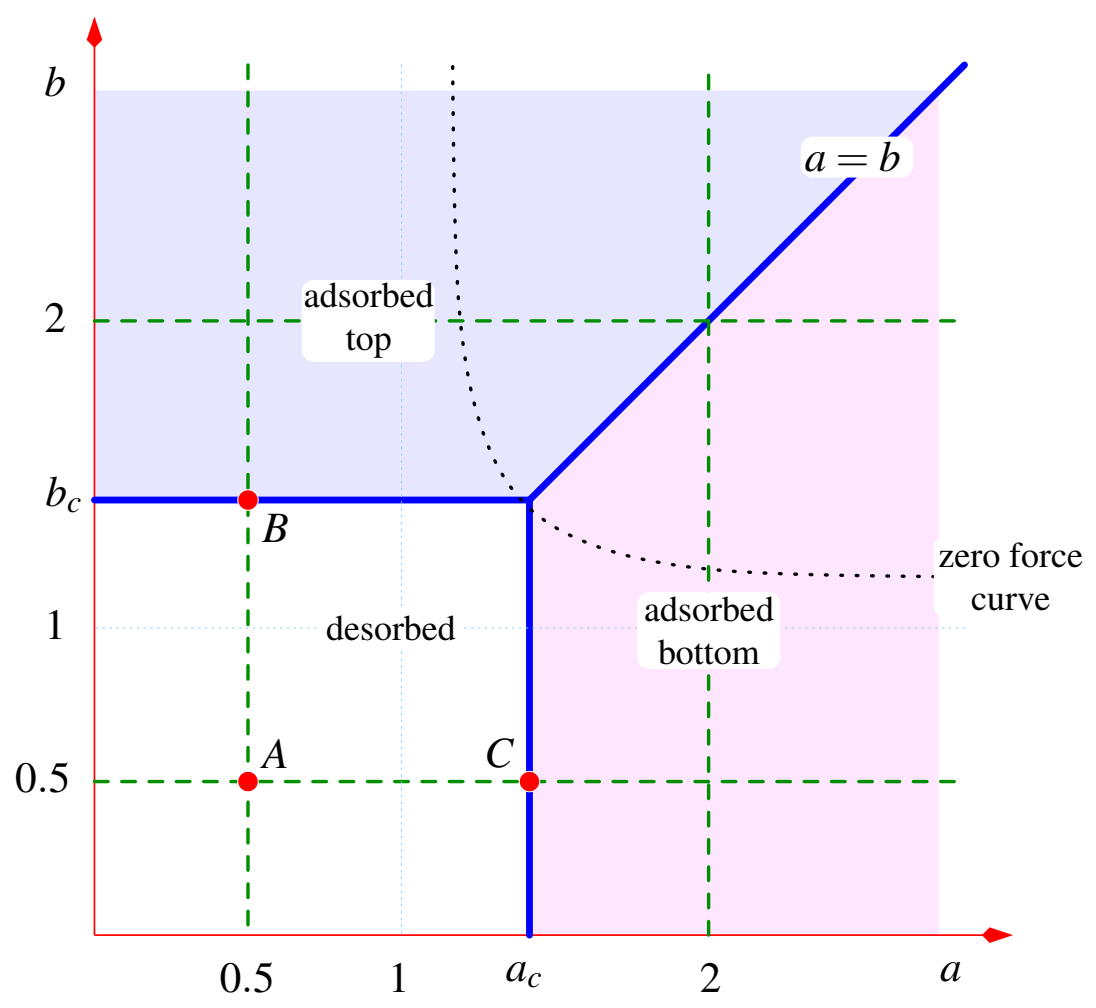

Figure 4. The conjectured infinite-slab phase diagram contains three phases in which the polymer is desorbed, adsorbed to the bottom surface and adsorbed to the top surface. The corresponding phase boundaries are indicated with solid lines. We have simulated the system along the lines $\{(a, 1 / 2),(a, 2),(1 / 2, b),(2, b)\}$ (indicated with dashed lines). The three points A, B and C are those at which we estimate the scaling function.

and each of the adsorbed phases. While the lines $(2, b)$ and $(a, 2)$ were chosen to study the transition between the two adsorbed phases. Our results confirm that the this transition is distinctly different in nature from the desorbed-adsorbed critical transitions.

Let us first consider the lines $(a, 1 / 2)$ and $(1 / 2, b)$ which cut through the transitions from the desorbed phase to the adsorbed phases which should occur at $a=a_{c} \approx 1.33$ and $b=b_{c} \approx 1.33$ respectively. In Figure 5 we show the fluctuation of contacts with the bottom surface for lengths 64, 128, 256 and 512 in the slab of width 20. We plot these quantities divided by the length of the polymer. We see that the peak heights of the fluctuations are growing weakly with $n$. Along with consideration of the distributions of contacts of the polymer with the walls which 
display a single peak (when considered at the parameter values of the specific heat peak) we conclude that this transition (in the infinite $w$ limit) is second-order with a crossover exponent near 0.5 (and so a specific heat exponent near 0) - we can provide no reasonably precise estimate of the crossover exponent however. This is in accord with the proposal that both the transitions are second-order transitions of the same type and being of the same type as the half-space adsorption transition. At width 20 we estimate the position of the pseudo-transition to be at $a=1.38(4)$ and we observe that the peaks of the specific heat move to lower values of $a$ as the width is increased: this is in accord with the estimates of the half-space adsorption transition at around 1.33 .

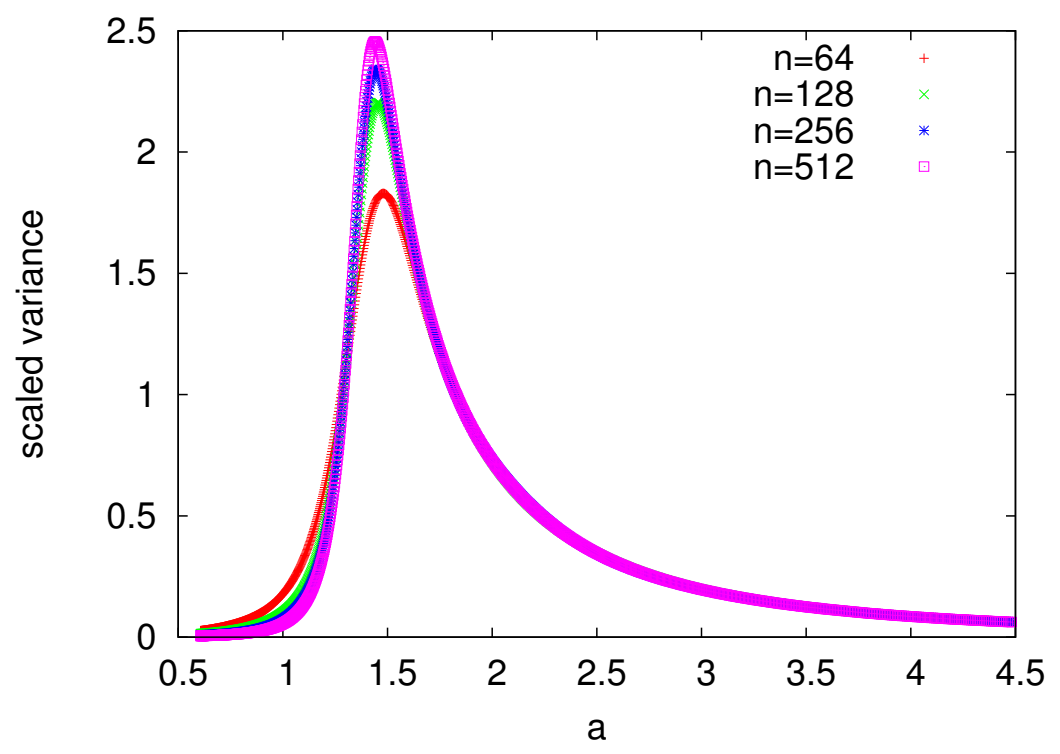

Figure 5. The variance of contacts with the bottom surface per length along the line $(a, 1 / 2)$ for width 20 and lengths $64,128,256$ and 512.

Consider now the lines $(a, 2)$ and $(2, b)$; these pass from one adsorbed phase to the other, cutting across the transition line $a=b$. We expect from the directed problem [4] to see a much stronger transition at approximately $a=2$ and $b=2$ respectively. We plot the fluctuations in the number of bottom surface contacts divided by length squared in Figure 6 . The fluctuations per length squared shows a sharp transition around $a=2$ and $b=2$ as expected: the convergence of the peak heights of the fluctuations divided by the square of the length indicate a crossover 
exponent of $\phi=1$, which in turn implies a specific heat exponent $\alpha=1$ (that is, a first order transition). To confirm the hypothesis of a first order transition we plot the distribution of the contacts with the bottom surface at the transition: this is shown in Figure 7, where we clearly see two peaks.

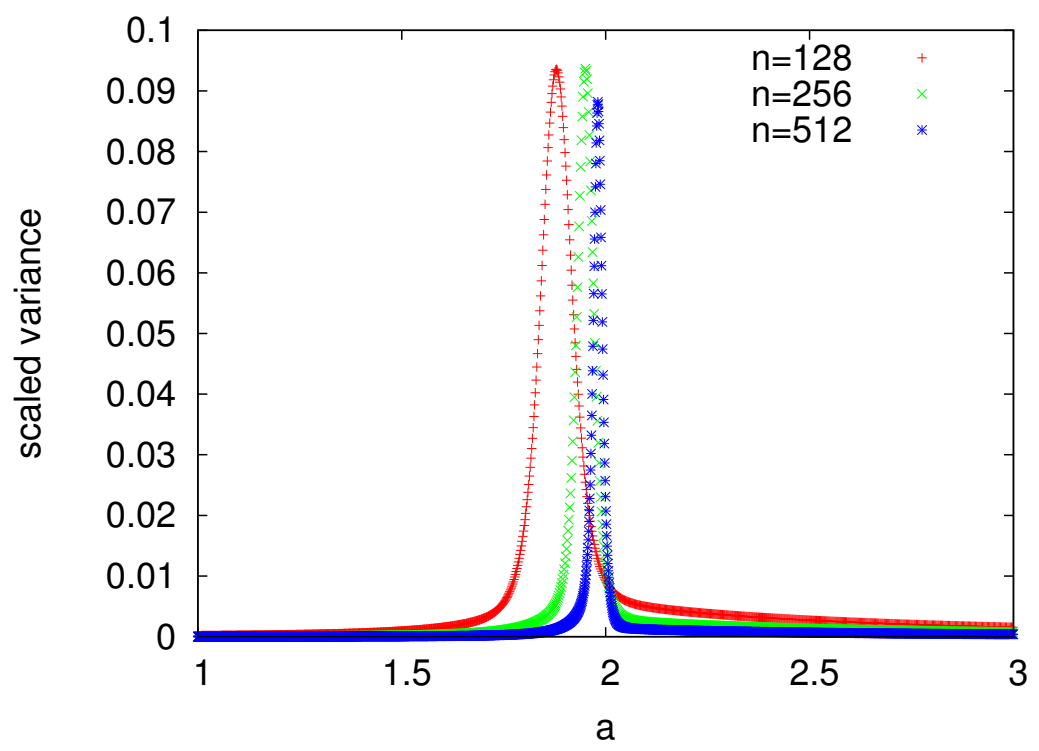

Figure 6. The variance of contacts with the bottom surface per length squared along the line $(a, 2)$ for width 12 and lengths 128, 256 and 512. Note that the peak height stays approximately constant implying that the peak height $h_{n} \sim n^{2}$.

Additionally, we have studied the scaling function of the free energy at three points in the $(a, b)$ plane: $(0.5,0.5)$ (point A), $\left(0.5, b_{c}\right)$ (point B), and $\left(a_{c}, 0.5\right)$ (point C) - see Figure 4. Since we do not have a precise estimate of $a_{c}=b_{c}$ on the cubic lattice we did not examine the point $\left(a_{c}, b_{c}\right)$. Ideally we would like to have studied the scaling function of the force, however this requires precise estimates of free energies at multiple widths and we found that would could not obtain sufficient precision within a reasonable time-frame with the resources available to us.

Using the conjectured scaling of the free energy given in equation (3.5), we have estimated $\mathcal{K}$ by plotting $n\left(\frac{\log Z_{n}(w)}{n}-\log \mu(3)-g \frac{\log n}{n}\right)$ against the scaling variable, $n^{\nu} / w$ (see Figures 8, 9 and 10$)$. We have used $\mu(3)=4.684, \nu=0.588$ and $a_{c}=b_{c}=1.33$. The value of $g$ used depends on which point is being plotted. For points A and B where $a<a_{c}$ we have used $g=\gamma_{1}-1=-0.32$ while for point $\mathrm{C}$ 


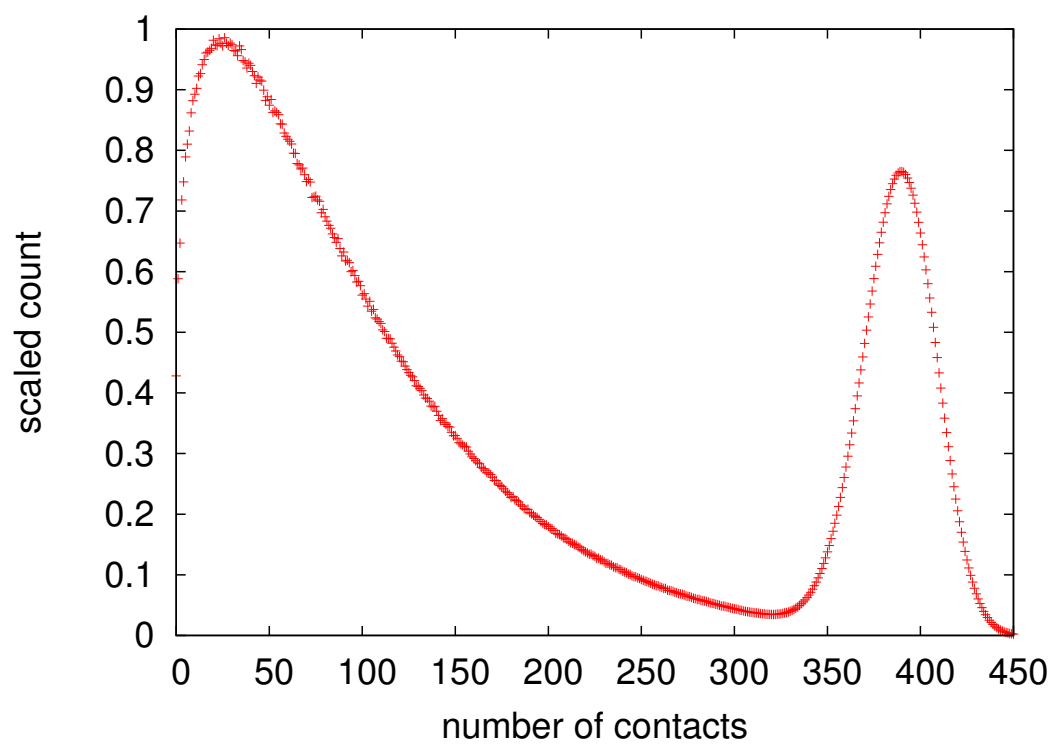

Figure 7. The distribution of contacts with the bottom surface from the simulations along the line $(a, 2)$ where $a$ was chosen to be at the peak of the variance of contacts with the bottom surface. The value of $a$ used was 1.975 , for data produced from simulations at width 12 and polymer length 512 .

we found that data collapse was best using an estimate for $\gamma_{1, s}-1=0.25$ which is just outside the value computed by DeBell and Lookman [18].

While $\mathcal{K}$ is monotonic at points $A$ and $C$, we find that it is distinctly unimodal at point $B$. We conclude that at points $A$ and $C$, the polymer exerts a repulsive force on the plates at all lengths and widths. Whereas at point $B$ we see that there is a combination of length and width such that the free energy has derivative (with respect to $w$ ) equal to zero.

At point $A$ the interactions with both confining planes are repulsive and the entropy loss due to confinement leads to a repulsive force. Point $C$ corresponds to a critical value of the attraction at the plane where the walk is tethered and there is no attractive force with the other plane, so the force is repulsive. At point $B$ the walk is tethered to one plane but attracted to the other. If $n \rightarrow \infty$ at fixed $w$ it is known rigorously that the force is repulsive (Janse van Rensburg et al 2006) and this corresponds roughly to the case where $n^{\nu} / w>>1$. If $n^{\nu}<<w$ the walk extends to allow vertices in the top plane and this leads to an attractive force. 


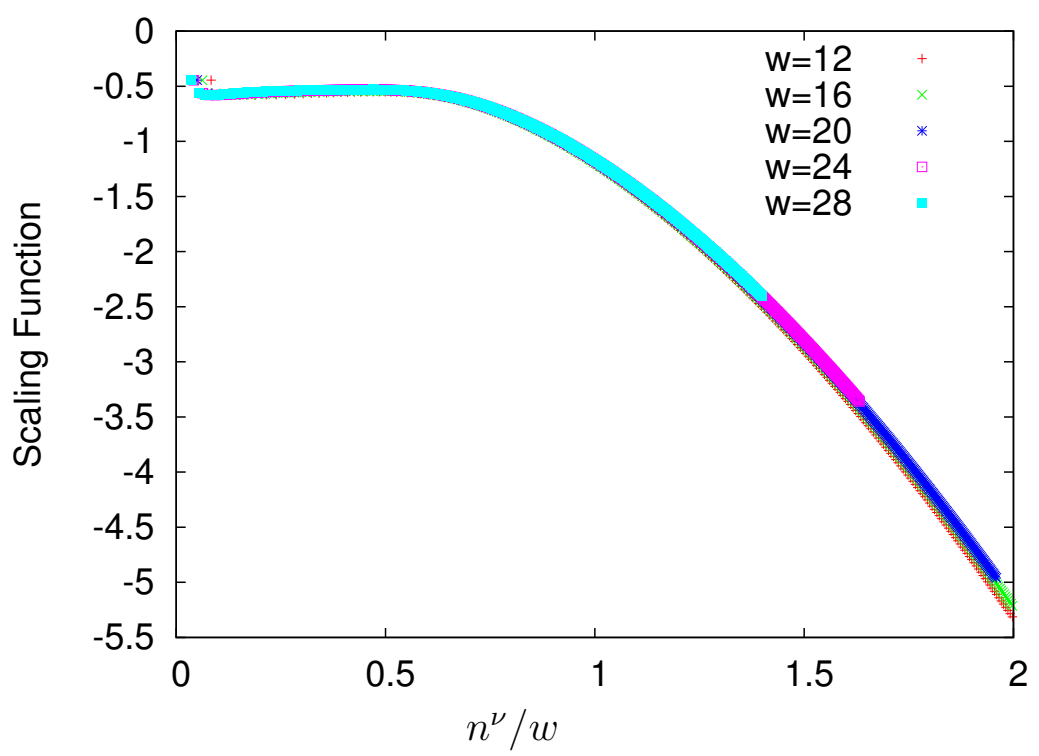

Figure 8. A plot of the scaled free energy at the point $A(1 / 2,1 / 2)$ for widths $12,16,20,24$ and 28 and lengths from 0 to 512. The horizontal axis is $n^{\nu} / w$ and the vertical axis is $n\left(\frac{\log Z_{n}(w)}{n}-\log \mu(3)-\left(\gamma_{1}-1\right) \frac{\log n}{n}\right)$. We have used the values $\mu(3)=4.684, \nu=0.588$ and $\left(\gamma_{1}-1\right)=-0.32$.

\section{Discussion}

We have examined the phase diagram and 'force-diagram' of a lattice polymer between two attractive plates. Additionally we have confirmed the existence of a finite-size scaling theory in the desorbed and critically adsorbed regions of the phase diagram (of the infinite slab). Intriguingly, while the numerical positions of the transitions and the bulk and half-space entropic exponents are different from the directed walk model solved on the square lattice [4] to this more "realistic" three-dimensional (presumably not exactly solvable) model the structure of the phase diagram, the order of the transitions, the general positions of the attractive and repulsive force regions, as well as even the shape of the scaling functions at corresponding points are essentially the same (numerical evidence for the directed case [21] confirm this, and an exact calculation of the scaling functions [22] is forthcoming). It has long been conjectured that the crossover exponent for the halfspace adsorption model is 'super-universal', being independent of the dimension (and whether directedness is imposed). Now it seems that the more complex problem of 


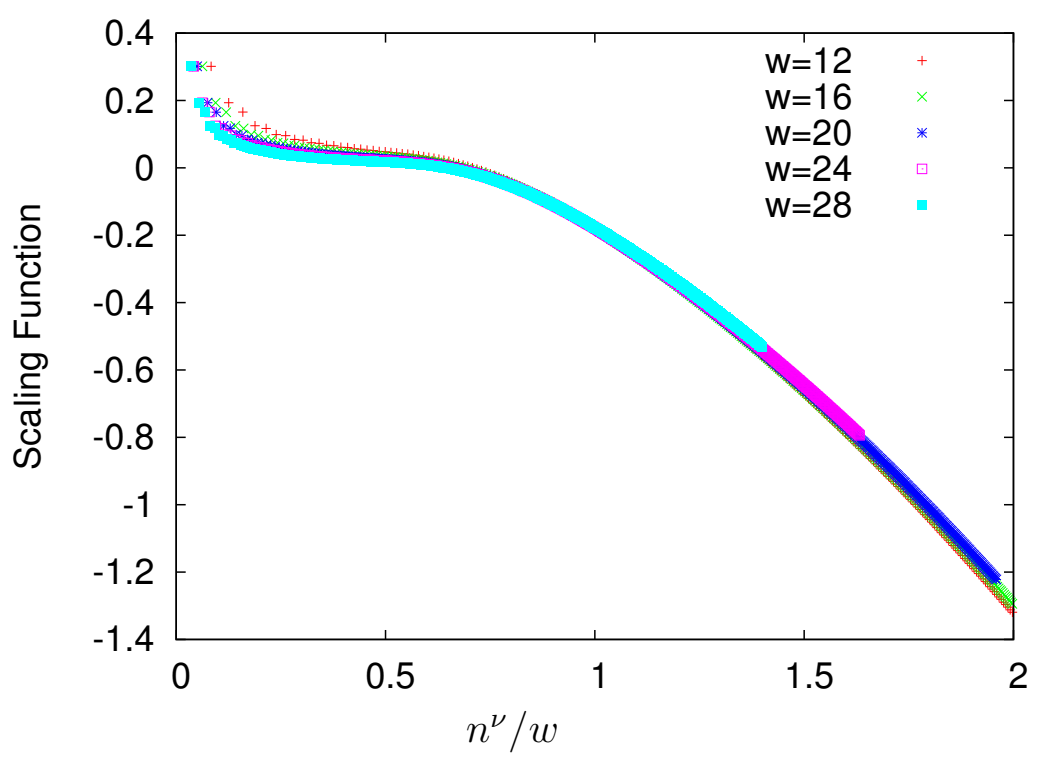

Figure 9. Plots of the scaled free energy at the points $C\left(a_{c}, 1 / 2\right)$ using $a_{c}=1.33$, for widths $12,16,20,24$ and 28 and lengths from 0 to 512 . The horizontal axis is $n^{\nu} / w$ and the vertical axis is $n\left(\frac{\log Z_{n}(w)}{n}-\log \mu(3)-\left(\gamma_{1, s}-1\right) \frac{\log n}{n}\right)$. We have used the values $\mu(3)=4.684, \nu=0.588$. We have used $\left(\gamma_{1, s}-1\right)=0.25$

a polymer in $d$-dimensional space confined between two $d-1$ dimensional surfaces demonstrates even more remarkable dimensional robustness. An explanation of these phenomena is terms of field-theoretical/renormalization group language would be interesting.

\section{Acknowledgements}

The authors would like to thank Gary Iliev and Buks van Rensburg for helpful discussions and the Australian Research Council (via its support of MASCOS) and NSERC of Canada for financial support.

\section{References}

[1] Bemis J E, Akhremitchev B B and Walker G C 1999 Langmuir 15 2799-805

[2] Napper D H 1983 Polymeric Stabilization of Colloidal Dispersions (London: Academic Press)

[3] DiMarzio E A and Rubin R J 1971 J. Chem. Phys. 55 4318-36

[4] Brak R, Owczarek A L, Rechnitzer A and Whittington S G 2005 J. Phys. A: Math. Gen. 38 4309-25 


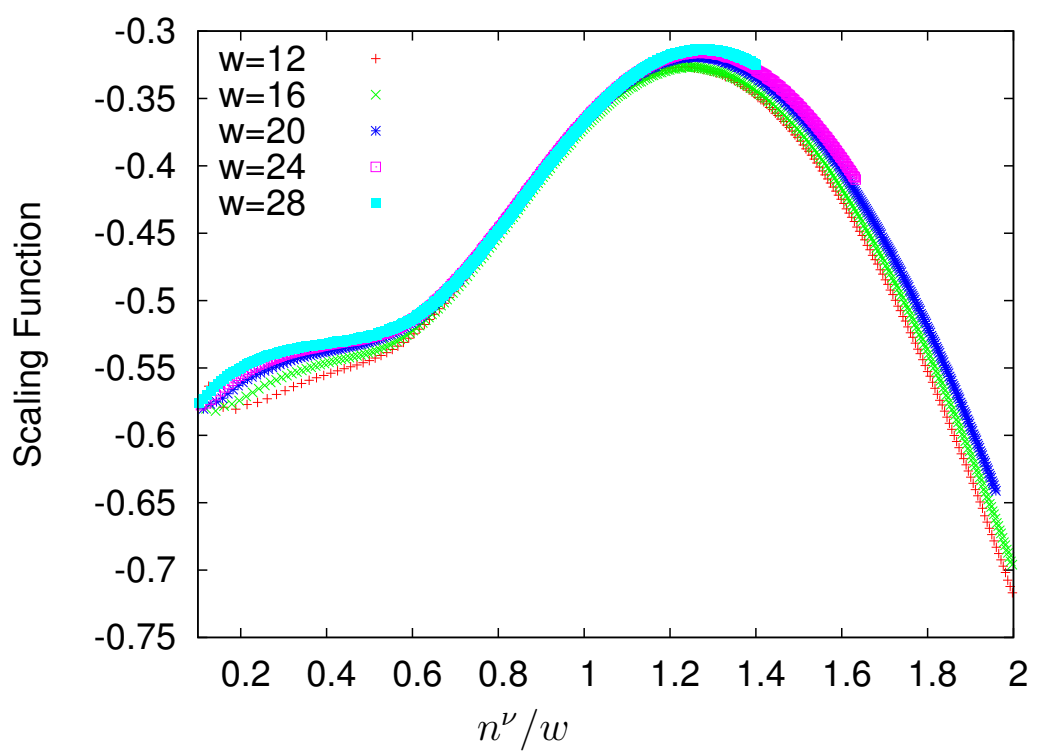

Figure 10. A plot of the scaled free energy at the point $B\left(1 / 2, b_{c}\right)$ using $b_{c}=1.33$, for widths $12,16,20,24$ and 28 and lengths from 0 to 512. The horizontal axis is $n^{\nu} / w$ and the vertical axis is $n\left(\frac{\log Z_{n}(w)}{n}-\log \mu(3)-\left(\gamma_{1}-1\right) \frac{\log n}{n}\right)$. We have used the values $\mu(3)=4.684, \nu=0.588$. We have used $\left(\gamma_{1}-1\right)=-0.32$.

[5] Wall F T, Seitz W A and Chin J C 1977 J. Chem. Phys. 67 434-8

[6] Klein D 1980 J. Stat. Phys. 23 561-86

[7] Alm S and Janson S 1990 Commun. Stat.-Stoch. Models 6 169-212

[8] Stilck J F and Machado K D 1998 Euro. Phys. J. B 5 899-904

[9] Stilck J F 1998 Brazil. J. Phys. 28 369-79

[10] Janse van Rensburg E J, Orlandini E, Owczarek A L, Rechnitzer A and Whittington S G 2005 J. Phys. A: Math. Gen. 38 L823-8

[11] Janse van Rensburg E J, Orlandini E and Whittington S G 2006 J. Phys. A: Math. Gen. 39 13869-902

[12] Hammersley J M and Whittington S G 1985 J. Phys. A: Math. Gen. 18 101-111

[13] Janse van Rensburg E J and Rechnitzer A R 2004 J. Phys. A: Math. Gen. 37 6875-98

[14] Daoud M and de Gennes P G 1977 J. de Physique 38 85-93

[15] Rechnitzer A and Janse van Rensburg E J 2002 J. Phys. A: Math. Gen. 35 L605-12

[16] Prellberg T and Krawczyk J 2004 Phys. Rev. Lett. 92120602

[17] Krawczyk J, Prellberg T, Owczarek A L and Rechnitzer A 2004 J. Stat. Mech. P10004

[18] De Bell K and Lookman T 1993 Rev. Mod. Phys. 65 87-113

[19] Hammersley J M, Torrie G M and Whittington S G 1982 J. Phys. A: Math. Gen. 15 539-571

[20] Nienhuis B 1982 Phys. Rev. Lett. 49 1062-1065

[21] Owczarek A L and Rechnitzer A unpublished, 2005 
[22] Owczarek A L, Prellberg T and Rechnitzer A manuscript in preparation, $200 \%$. 